\title{
Quality of Service based Multicasting Routing Protocols for MANETs: A Survey
}

\author{
Prabhat Kumar Sahu* ${ }^{1}$, Binod Kumar Pattanayak ${ }^{2}$ \\ Faculty of Engineering, Siksha 'O' Anusandhan University \\ Bhubaneswar, Odisha, India \\ *Corresponding author, e-mail: prabhatsahu@ soauniversity.ac.in
}

\begin{abstract}
The modern electronic age consists of many new approaches for the communication among the human civilizations and the $A D$ HOC network is one of the successful self-configuring and infrastructure-less communicating approach. Mobile ad hoc networks (MANETs) comprised of mobile nodes organize themselves in such a manner that the can move freely inside the network and frequently change their position. Open architecture and dynamic nature of the network enhance its use in multimedia applications that needs graphics, audio, data, image, video and animation. Now a day's the increasing use of multimedia and Internet technology needs quality of service $(Q o S)$ in MANETs in order to provide better service for the companies. This type of applications lends themselves well to multicast operations. As multicasting supports group oriented computing it can improve the QoS of the wireless medium by means of sending multiple copies of packets by exploring the internet broadcast properties of wireless transmission. Hence QoS multicasting plays a great role in MANETs for multimedia applications. However it is very difficult and challenging task to provide QoS multicasting. With unique features and by means of different recovery mechanisms, many researchers have been proposed various QoS based multicasting routing protocols for MANETs. In order to assist QoS multicasting routing protocols design for MANETs, we characterize the taxonomy of the multicast routing protocols and design features in this paper.
\end{abstract}

Keywords: MANETs; QoS; multicasting; routing protocols

Copyright (C) 2017 APTIKOM - All rights reserved.

\section{Introduction}

Mobile Ad hoc Networks (MANETs) are a class of communication networks of mobile devices which are self-configuring and inter-connected to each other with wireless links. Basically, the networks are without a fixed infra-structure i.e. the devices are mobile and independent. The MANET has proved its importance in on line education, emergency operations, disaster relief operations, etc, wherever multipoint-to-multipoint communication becomes a requirement. An efficient way to utilize multipointto-multipoint communication is multicasting. MANETs usually do not provide QoS (Quality of Services) guarantees and reliable ser-vices. The main sources of unreliability are limited memory and processing power, limited capacity of battery, lesser stability under high mobility and unpredictability of nodes. Parameters like bandwidth, delay, packet loss and jitters are specifically needed to be taken care of while increasing the reliability and stability of MANETs.

Services like QoS and multicast have become a major requirement due to the increase in the demand of multimedia applications in the mobile market. Multicasting is an efficient technology which supports one-to-many or many-to-many bandwidth-dedicated communications. During multicast communication, the sender node sends only a single packet with the group address as the destination, after which the network replicates the packet to reach all the available destinations. This results in high scalability and more bandwidth savings. In this paper, a summarization of several multicast routing mechanisms in MANETs that can help the scientific and research community to work towards further development in the per-existing mechanisms is presented. The paper is organized as follows. Section 2 describes the multicasting routing for MANETs. Section 3 describes QoS in MANETs. Section 4 describes the Taxonomy of QoS based multicasting routing protocols. Section 5 describes Proactive routing strategy based multicasting routing protocols. Section 6 describes reactive routing strategy based multicasting routing protocols. Section 7 describes hybrid routing strategy based multicasting routing protocols. Section 8 describes Cluster-based multicasting routing Protocols. Section 9 describes 
evaluation and comparison of protocols in terms of structure attributes and performance metrics. Section 10 concludes the paper.

\section{Research Method}

Now a day's multicast communication is widely used as the data packets are send from one source node to multiple number of destination nodes without replicating the data packets at the source node rather than it create duplicate data copies in the network. As a result, bandwidth consumption becomes low and the communication cost gets reduced and throughput becomes low and power consumption becomes high. The advantages of multicast routing is for proficient savings of bandwidth, saving of network resources as the sender node an send the same data with a single transmission to multiple number of receiver nodes, communication cost is reduced and network load is minimized.

\section{QoS in MANETs}

It is a very difficult task for the researchers because the topology of an ad hoc network constantly changes which is very challenging. QoS refers to a set of services that are provided by the network while transmitting packets from a source node to destination node. There are various parameters of QoS that are guaranteed by the network to the users such as delay, bandwidth, probability of packet loss, delay variance (jitter), power consumption etc. Previously ad hoc network was designed for only army use, but now because of its ease of mobility \& flexibility it is made available to private citizens use also. With the increase in demand the quality of service \& its performance is now expected by the users of wireless network. Factors that influence QoS of Ad hoc network are:

1. Low throughput:-It represents the total number of bits transmitted by each node to its destination in a wireless network. Due to more data loads from users sharing the same message data the bit rate/throughput provided to the data stream may be too low.

2. Dropped packets:-The routers may fail to deliver some packets if the data loads are corrupted or if the network fails due to some problem. At this time the data is again retransmitted causing severe transmission delay.

3. Errors:-In wireless communication due to bit errors caused by noise and interference some packets are corrupted.

The parameters of QoS include bandwidth, delay, jitter, security, and network availability \& battery life \& packet loss. The most important QoS metrics are delay, jitter, loss, \& throughput. Delay is the time between the arrival of the packet $\&$ successful delivery of the packets to its desired destination. Jitter is the variation of delay for the transmission of packets \& it's an important parameter for multimedia application of ad hoc network. Bandwidth is the measure of a capacity that the data can transmit from one node to another. Issues of QoS are present in robustness, routing protocols with multihop ad hoc network. QoS area in this wireless ad hoc network is still an unauthorized area. The goal of QoS in MANET is to achieve a more deterministic network behavior so that the data carried by the network can be successfully delivered and network resources can be well utilized.

\section{Taxonomy of Qos Based Multicasting Routing Protocols}

Appropriate classification methods are needed in order to compare and analyze mobile ad hoc network routing protocols. Classification methods help researchers and designers to go through distinct characteristics of a routing protocol and find its relationship with others. So in this survey, we represent different QoS based multicasting routing protocols characteristics which are used to group and compare different approaches. Basically these characteristics are related to the information which is exploited for routing, when this information is acquired and the roles which nodes may take in the routing process. QoS based multicasting routing protocols are basically classified into following categories:

1. Proactive routing strategy based multicasting routing protocols

2. Reactive routing strategy based multicasting routing protocols

3. Hybrid routing strategy based multicasting routing protocols

4. Cluster-based multicasting routing Protocols 


\section{Proactive Routing Strategy based Multicast Routing Prtocols \\ 5.1. Multicast Core-Extraction Distributed Ad Hoc Routing (MCEDAR)}

There has always been a need for a multicast routing approach that has the combination of the advantages of both the tree based and the mesh based routing protocols. In [1], Prasun Sinha, Raghupathy Sivakumar and Vaduvur Bharghavan have proposed MCEDAR (Multicast Core-Extraction Distributed Ad Hoc Routing) that addresses all the issues of robustness and efficiency in one framework. This protocol comprised of two CEDAR's components basically the core and the core broadcast, a mesh-based routing structure that tries to offer an increase in reliability along with rise in the mobility of the network, a forwarding protocol based on tree, which computes the overhead generated by optimal forwarding in terms of delivery time of each data packet, elimination of creation of divisions in the mgraph when the network lying below is not divided and a pruning method that automatically prunes the mgraph. It depends on the main broadcast mechanism for forwarding of control packet and data, and therefore does not use the local broadcasts in its key mechanisms, disengages the control infrastructure from the data forwarding infrastructure resulting in lesser control overhead and higher efficiency of data forwarding.

\subsection{EQMGA - Entropy-based Genetic Algorithm to support QoS Multicast Routing}

In [2], Hua Chen, Baolin Sun and Yue Zeng have proposed a method to select optimized fuzzy controller parameters by the use of Genetic Algorithm. For mobile nodes which need intercommunication, Multicast ad hoc on-demand distance-vector (MAODV) routing protocol offers an efficient route establishment. Control overhead is minimum as MAODV is specifically designed for ad hoc wireless networks. The basic idea of using EQMGA algorithm is to build the new metric-entropy and select the long-life path by using entropy metric to have less number of route reconstructions. This offers QoS guarantee in the ad hoc network which changes its topology continuously. The chromosomes of the genetic algorithms contain integral queuing and a method which encodes according to routing representation, which is the common method of representation. The one with at-par performance has the highest level of fitness, and the one with below-par performance has the lowest level of fitness. Selection operation is used and many sub-individuals are produced by the selected individuals. After simulation, the results indicate that the mentioned approach and its parameters provide an exact and most efficient method of estimation and evaluation of stability of the route in dynamic mobile networks.

\subsection{HQMGA - Hierarchical QoS Multicast routing using GA in MANET}

The existing methods on QoS Multicast Routing have not been developed to compute multicast tree which satisfies multiple QoS constraints simultaneously. In [3], Eiichi Takashima, Yoshihiro Murata, Naoki Shibata, Keiichi Yasumoto and Minoru Ito have proposed HQMGA (Hierarchical QoS Multicast routing using GA in MANET) which permits MANET nodes to construct a semi-optimal multicast tree dynamically which can satisfy multiple QoS constraints simultaneously. The genetic algorithm (GA) is used to compute semi-optimal multicast tree because GA can quickly recompute a new multicast tree and can solve combinatorial optimization problem. All the nodes in MANET are segregated into numerous clusters like Open Shortest Path First (OSPF). For reduction of costs for collecting information on topology and computation of trees, sub-trees are figured out for these clusters. Multicast tree, the tree containing the local trees and the global tree is used. The method fulfils the objective i.e. minimizing the total power consumption for the construction of an optimized multicast tree.

\subsection{EGA - A Genetic Algorithm for Energy-Efficient Based Multicast Routing on MANETs}

Due to lack of balance in consumption of battery energy, mobile nodes face problems with energy overhead. In [4], Yun-Sheng Yen, Yi-Kung Chan, Han-Chieh Chao and Jong Hyuk Park have proposed a Genetic Algorithm for Energy-Efficient Based Multicast Routing (EGA). A genetic algorithm (GA) is a type of searching algorithm which with the use of genetic operators, emulate the idea of evolution by using natural selection and the concept of "survival of the fittest". Crossover is a genetic operator which carries out gene exchange from two chromosomes. By modifying some of the genes in random fashion, mutation (a genetic operator) enlists new gene structures into the population. A multicast spanning tree can be re-built by minimizing the delay constraint which in return results in extension of the lifetime of multicast service. Moreover, only mobile nodes with greater amount of energy can share the total energy of the multicast tree. To manage the outgoing node degree and to simplify the genetic operation, a topology encoding method and an extended sequence is used. The proposed method reduces the degree of the nodes with replacing a node having lower energy with a node possessing higher energy. Therefore, by the use of higher residual battery energy (RBE) at nodes, the distribution of the total battery energy consumption for the multicast service is carried out. Mutation for the repair function exchanges

Quality of Service based Multicasting Routing Protocols for MANETs: A Survey (Prabhat Kumar Sahu) 
leaf and core nodes. Each generation is reproduced from both crossover and mutation operators. Eventually, battery energy, reduction in convergent time and the maximum lifetime of multicast service are achieved.

\subsection{MACO - Ant Colony Algorithm Based on Orientation Factor for QoS Multicast}

In [5], Hua Wang and Zhao Shi have proposed a modified ant colony algorithm (MACA), containing combination of both the ant colony algorithm and the location-aided routing algorithm with the addition of orientation factors which represent location information. GPS presents the location information which is used to adjust the direction of search of the ant colony. The algorithm converges earlier due to bigger network topology scale resulting in local optimization. In bigger ant colonies, it becomes difficult to find quickly a better path from the large number of paths in a mess. The orientation factor is introduced while the probability of next hop is being computed. The probability of selecting the directed arc directing the destination node should be more with the help of the factor. The factor should also influence the next hop selection. Besides the inclusion of the orientation factor, the tabu search and simulation annealing are integrated resulting in speeding up of convergence and avoidance of the search result leading to local optimal solution.

\section{Reactive Routing Strategy Based Multicast Routing Protocols 6.1. QoS-Aware Minimum Energy Multicast (QoS-Mem)}

Wireless nodes always depend upon limited battery source. The routing protocol for provisioning of QoS should take into consideration the remaining battery power and the battery consumption rate for increasing lifetime of such networks. Bandwidth is considered as the only QoS parameter and a constraint formulation model is proposed for the QoS-aware Minimum Energy Multicast (QoS-MEM) as proposed by authors in [7]. The issues of minimization of energy consumption for the purpose of broadcasting and multicasting are generally explained in detail by the MEB (Minimum-Energy Broadcast) problem and MEM (Minimum-Energy Multicast) problem. For the MEB problem, the broadcast trees use a straight greedy approach that contains the best unicast paths to every individual destination from the source node. A source-initiated multicast uses a tree-like structure in the network for delivering the same data stream to a group of receivers efficiently. For receiving signal and processing activities, it is assumed that there is no power consumption involved. In return, for transmission at each node, total power is utilized. For being a bandwidth-constrained multicast tree (BCMT), two constraints need to be satisfied: Rooted Tree Constraint and Bandwidth Constraint. The conditions reflected by the bandwidth constraints are that the allocated bandwidth on each link of the multicast tree must meet the required bandwidth and must be free from any conflicts.

\subsection{On-Demand QoS Multicast for MANETs (ODQMM)}

On-Demand QoS Multicast for MANETs (ODQMM) protocol is proposed by authors in [8] that offers reservation of bandwidth for multicast groups ensuring availability of bandwidth information from the beneath layer such as in TDMA network. A node can bond with a multicast group acting as both data source and receiver. For indication about requirement of resource reservation, new flags are introduced into the Route Request (RREQ) message. The RREQ message encapsulates the bandwidth amount requested. Similar amount of bandwidth is reserved by the nodes of multicast tree as per sender's request. ODQMM naturally offers two reservation styles: fixed-filter (FF) and shared-bandwidth filter (SB). A unique reservation is done for data from each source in fixed-filter and it is ensured that the remaining senders in similar session do not share the reserved resources. A single reservation is shared by flows from all senders in the similar session in shared-bandwidth filter. Extension of the Group Hello message, which is generally used in MAODV, is done by ODQMM with the inclusion of the information on the entire reserved bandwidth under FF and SB. Individual nodes running ODQMM are required to maintain four tables: Reservation Table, Route Table, Multicast Route Table and Group Leader Table. Normally six control messages are used namely RREQ, Route Reply (RREP), Group Hello (GRPH), Multicast Activation (MACT), Keep Alive and QoS Error. Whenever a node tries to find a route to a multicast group, route discovery cycle takes place. Detection of link breakage occurs when there is no reception of packets from its neighbour after a fixed time interval.

\subsection{Call- Admission Multicast Protocol for MANETs (M_CAMP)}

M-camp, a reactive multicasting routing protocol provide a mechanism to ensure bandwidth estimation for multicasting in wireless ad hoc network for the admission control of multimedia and the

APTIKOM J. CSIT Vol. 2, No. 1, 2017 : $31-46$ 
system configuration in MANETs [6]. AS it supports group-orientated mechanism that operates on per to per call, it is independent of underlaying wireless technologies. There's absolutely not required any maintenance of status information of mobile hosts in the network. In order to evaluate the bandwidth availability in the network between the source and a group of destinations, it adopts measurement based approach and ensures scalability. It ensures scalability and energy saving as the mobile hosts are responsible for forwarding the QoS traffic in the network.

\subsection{QoS Ad Hoc on-Demand Distance Vector Routing (QoS-AODV)}

QoS-AODV, an on-demand routing protocol is proposed in [9] which finds routes to the destination which are discovered when needed. A timer-based state in each node is maintained by AODV. For the above discussed QoS-AODV protocol, delay is considered as a QoS constraint. The QoS functionality is implemented to deal with the finite resources available in an active environment which specifies a metric for such mobile wireless networks. Each node includes the creation time in RREQ messages while process of discovering the neighbour is being carried out by the QoS-AODV. The measured delay is the difference between the noted time and the current time which is computed when a neighbour node receives this message. One-way measured delay consists of the collision avoidance time, the control overhead time, the queuing time and the transmission time. Otherwise, the rise of traffic load at the addition of acknowledgment messages to the QoS-AODV protocol is avoided while measuring the one-way delay. Generally, any rise in mobility does not affect QoS-AODV. Therefore, use of multicasting results in decrease of communication costs for applications that send similar data to more than one recipients. It also results in minimization of sender and router processing and delay in delivery.

\subsection{Ad Hoc QoS Multicasting (AQM)}

Ad Hoc QoS Multicasting (AQM), proposed in [10], offers QoS support and multicasting features to ad hoc networks. With the help of resource management, it achieves the efficiency of multicasting. Based on previous reservations, AQM checks for availability of bandwidth within each node's neighbourhood and makes it sure that latest QoS information is used to chose routes. The main criterion for evaluating AQM is ratios of service satisfaction which are best described both at member and session levels. Improved satisfaction grades and efficiency in multicasting for members and sessions is achieved by AQM with the application of QoS restrictions to the ad hoc network. AQM constitutes flat and simple network structure, in which all nodes are equal. It avoids complex network topologies which are difficult in terms of design and maintenance. Sessions of cluster to cluster multicast are managed by AQM, whereas communications within the cluster are handled by a higher layer. A hybrid method is proposed by AQM with the help of multicast routing with table-driven session management and during the starting of a join process, on-demand verification of QoS information is carried out.

\subsection{Quality of service for Multicast in MANETs (QAMNet)}

Quality of service for Multicast in MANETs (QAMNet), a mechanism for improvisation of Quality of Service (QoS) for multicast communication in MANETs, is detailed in [12]. Mesh based multicast routing protocols have been extended by the inclusion of traffic privatization, distributed resource probing and admission control mechanisms and adaptive rate control of non-real-time traffic according to the Medium Access Control (MAC) layer feedback for maintaining low delay and throughput for real-time multicast flows. Mesh based multicasting delivery structures are robust and versatile. Robustness is achieved along with high overhead because of redundant routes. It leads to early saturation of network in case of high traffic. Throughput rises with increasing traffic load, till the load reaches network capacity and consequently delay increases. Therefore, a need arises for load controlling for real-time multicast traffic so that it stays below network capacity in order to manage end-to-end delay. QAMNet offers distributed resource probing along with admission control mixed with the process of mesh creation. Controlling of local traffic for non real-time flows for minimization of the delay of realtime packets is carried out. Signalling packets developed for creating and maintaining mesh are recycled. The delay and packet loss rate of multicast real-time packet is reduced by 50 milliseconds over the complete range of mobility of the nodes and thus, a reduction in overhead is also seen.

\subsection{Cross-layer QoS Multicast Routing Protocol (E-QMR)}

QoS Multicast Routing (QMR), a protocol based on mesh topology, uses on-demand connection of group members and offers QoS paths for multicast groups [13]. The QMR protocol dissolves reservation function of bandwidth with multicast routing protocol taking into consideration that the remaining bandwidth is constant and equal with the raw channel bandwidth. QMR includes methods that

Quality of Service based Multicasting Routing Protocols for MANETs: A Survey (Prabhat Kumar Sahu) 
offer hybrid fix-reservation and shared-reservation bandwidth for securing QoS multicast routing. For preventing intermediate node from being overloaded and rejecting requests from new sources, admission control is put forth. The network layer must communicate with the MAC layer to compute the remaining bandwidth, in order to take a correct decision to accept or reject a new request. Estimating the remaining bandwidth with the help IEEE is still impossible due to sharing of bandwidth between neighbouring nodes. Estimation of remaining bandwidth is done according to the channel status of the radio and the computation of the periods, which are generally idle, of the shared wireless media. While estimating the available bandwidth, the activities of the neighbouring nodes must be taken into account.

\subsection{Secure Efficient QoS Multicast Route Discovery for MANETs (SEQMRAN)}

The affected nodes that may involve in modification or disruption of the sequential exchange of packets and taking wrong directions in routing, may act as a threat to the trust relationship. This ensures the security in QoS multicast routing protocol. Security of ad hoc routing protocol is normally dependent upon symmetric cryptography. Secure Efficient QoS Multicast Route Discovery for MANETs (SEQMRAN), proposed in [14], depends upon a one-time signature mechanism named as HORSEI which includes most reliable signing and verifying. The aim is to offer authentication to source for various QoS multicast routing discovery schemes. The protocol is setup of three main phases - 1) connection to a secure QoS multicast group, 2) creation of and 3) connection to a secure QoS multicast tree. Verification process is carried out in which the membership of the requesting node is counter-checked by Universal Resource Sharing Application (URSA), no matter who has sent the request. To communicate within a group securely, a secure QoS multicast session is initiated by the group member, i.e. creation of a secure QoS-guaranteed multicast tree and building up of secure and verified routes.

\subsection{Framework for QoS Multicast (FQM)}

A cross-layer design using a new QoS multicast routing protocol is proposed by authors in [15] that supports QoS multicast applications in MANET. The QoS route request must enter into the admission control module and based on the available bandwidth information, it is either accepted or rejected. The control and RT (Real-time) packets bypass the shaper and are sent directly to the interface queue. BE (Best-effort) packets must enter the shaper and are regulated according to the dynamic rate control. This protocol is a flexible hybrid scheme that is a combination of features from both IntServ and DiffServ. First, the forward node provides IntServ to for every source for the QoS route request that has been accepted. Second, the forward node provides DiffServ when data packets are received from other sources, in case of extra bandwidth. Here, usable bandwidth is divided into fixed-bandwidth for sources with route request entries, and shared-bandwidth for all other sources. The features of this protocol consists of efficient use of remaining bandwidth, reduced control overhead by dropping route requests and reduced redundant data transmissions. The cross layer design uses the passive listen method which is the best way to compute the available bandwidth with minimal control overhead. Regulating BE traffic based on dynamic rate control improves the performance of RT traffic and gives BE traffic opportunity to use the residual bandwidth efficiently.

\subsection{QoS On-Demand Multicast Routing Protocol (QoS-ODMRP)}

The features such as dynamic network topology, lack of exact state information, lack of central controller, limited resource availability, hidden terminal problem, impose challenges to ad hoc networks. On-Demand Multicast Routing Protocol (ODMRP), proposed in [16] that uses on-demand routing techniques with the implementation of a forwarding group where a set of nodes forward multicast data on shorter paths between any pair of members. The source is responsible for on-demand updating and establishing the group membership and multicast routes. A multicast source leaves a group, by simply stopping to send the join-request and when a receiver doesn't want to receive anymore and then it removes the entries from the member-table. For providing QoS requirements along a path, the admission policy must ensure that the minimum requested bandwidth is achieved for each flow. To determine enough bandwidth, we should have knowledge about the available link capacity and the bandwidth to be used by the requesting flow. Multicast protocols having more than one downstream node cannot be used to determine the consumed bandwidth. Information about neighbourhood is very much critical for QoSODMRP since it gives knowledge about the mobility, traffic and local topology. Similar to ODMRP, when a source has to send a data, it adds the requested bandwidth to the join-request packet and then floods it into the network. When the remaining bandwidth is more than the used bandwidth and also more than the minimum requested bandwidth, a new session is added to the reservation table and the session is

APTIKOM J. CSIT Vol. 2, No. 1, $2017: 31-46$ 
set to 'explored'. QoS-ODMRP results in increasing performance of the network in comparison with ODMRP by reduction of overhead and efficient utilization of bandwidth.

\subsection{QoS aware Multicast Routing Protocol (QMRP)}

QoS aware Multicast Routing Protocol (QMRP) elects the efficient route according to Forwarding Count and Non-Forwarding Count [17]. When a source node requires sending data to receiver nodes, a multicast mesh is constructed consisting of source, receiver, forwarding nodes and links connecting them. A combination of source address, sequence number and multicast address collectively identifies the RouteRequest packet in a unique way. The receiver node on reception of RouteRequest packet updates its RouteRequest cache and thus, broadcasts a RouteReply packet. The routine work of a forwarding node is to broadcast Data, OndemandMaintenance, or PeriodicMaintenance packets again immediately after reception and the purpose of the nearby node is to rebroadcast OndemandMaintenance or PeriodicMaintenance. QMRP is constructed such that each individual node regularly broadcasts the hello message. QMRP should have knowledge about the bandwidth consumption by the immediate neighbours. An upstream node after reception of the RouteReply packet updates the maximum bandwidth in its memory. QMRP initiates the maintenance process only after confirmation of non-reception of hello message by the forwarding node. QMRP uses this process to avoid maintenance routes that are not necessary and to reduce overhead.

\subsection{Multiple Paths/Trees (MPT)}

The QoS multicast routing is a new mechanism utilizing various parallel paths or trees to achieve the bandwidth requirement and the delay within the required delay bound. This mechanism can reduce the probability of system blocking as it is distributed and the route discovery and route reply stages follow the exact method as the traditional on demand routing protocols. The Code Division Multiple Access (CDMA) is overlying on the top of the Time Division Multiple Access (TDMA) structure. Each node in CDMA implements a before-assigned code to communicate with nearby nodes in an error free fashion. After the completion of route discovery and reply phases, construction of multiple paths/trees for multicast routing is done by selecting suitable paths or trees. The aim is to minimize the network cost. Authors in [18] follow three strategies: shortest path tree based multiple-paths (SPTM), least cost tree based multiple-paths (LCTM) and multiple least cost trees (MLCT), each having a different objective. The SPTM method minimizes the delay from source to destination. The LCTM minimizes the network cost. Various paths added to the SPT or LCT help the multicast connection in achieving the bandwidth requirement. The MLCT method is used to search for multiple LCTs thus minimizing the network cost. The bandwidth requirement is thus satisfied and the multicast connection is realized by the union.

\subsection{QoS-Multicast Ad Hoc on-Demand Distance Vector Routing (QoS-MAODV)}

For establishing consumed channel resources like bandwidth for nodes, there are some mechanisms for unicast routing, but not for multicast routing. As proposed by authors in [19], the remaining bandwidth is computed based on the information received from nearby nodes in control messages. QoS-Multicast Ad Hoc On-Demand Distance Vector Routing (QoS-MAODV) is a tree-based multicast routing protocol based on MAODV protocol which constructs the routes on-demand and creates the shared trees. For prevention of intermediate nodes from being overloaded, admission control is implemented. The intermediate node rejects RREQs of new sessions in case of non-availability of bandwidth. For every multicast group, the tree consists of members of two different classes of nodes: the ones which joined the multicast tree and the ones which didn't join but still are forwarding. QoSMAODV protocol uses six different types of messages for creating QoS multicast tree: Route request (RREQ), Route reply (RREP), Multicast activation (MACT), Group hello (GRPH), Hello and QoS-lost. A multicast source requiring a route to a group, broadcasts a RREQ message with the required bandwidth set on bandwidth field, sets the join flag to true, and sets multicast group address on destination address field. A member of multicast tree holding a current route to the destination and even amount of bandwidth replies to the request by sending an RREP mlessage. The source node after reception of the RREP message stores the required bandwidth as temporary-reserved and computes the end-to-end delay of each individual path. If the source node receives many RREPs from various paths for its RREQ message, it selects the best path with lesser end-to-end delay and the minimum hop-count to group leader. Then, a multicast activation (MACT) message is sent which is used to activate the selected path from the source to the destination node sending the RREP. To maintain connectivity among the group, the group leader broadcasts group hello (GRPH) messages frequently. After setting up of such a route, if any node detects that the requested QoS parameters can no longer hold, then a QoS-lost message is originated back.

Quality of Service based Multicasting Routing Protocols for MANETs: A Survey (Prabhat Kumar Sahu) 


\subsection{Hexagonal-tree TDMA-based QoS multicast protocol (HTQ)}

A new TDMA-based QoS multicast routing protocol (hexagonal-tree QoS multicast protocol) for a wireless mobile ad hoc network is proposed in [20]. Attempts have been taken to construct a new multicast tree structure, denoting the hexagonal-tree for serving as the QoS multicast tree in which the MAC sub-layer adopts the TDMA channel model. Both the hidden-terminal and exposed-terminal issues are considered for exploitation of the time-slot reuse capability. The sub-path of a hexagonal-tree is a hexagonal-path. A hexagonal-path is specially a two-path structure. This results in improvisation of success rate with the use of multi-path routing. The efficiency of wireless sensor networks may gain from this proposed concept.

\subsection{Bandwidth-Aware Multicasting Protocol ( $\mathrm{Hu})$}

Constructing a multicast tree is easier than sending the same packet from the server to each client individually in case of multicasting packets. Limitation of bandwidth and lbattery power in MANETs should be taken into consideration for routing/multicasting protocols. By decreasing the number of hosts taking part in forwarding of packets, bandwidth and power consumption can be reduced. A bandwidth-aware multicasting protocol for MANET that determines a bandwidth satisfied multicast tree to create a flow of the requested bandwidth, is proposed in [21]. The increment in bandwidth resulting from the new flow depends upon selection of the host and its neighbours, as the forwarders for the flow. The remaining bandwidth of a host is estimated by controlling the amount of channel time which is idle. The algorithm, which provides a feasible solution to Bandwidth-Satisfied Multicast Trees Problem, can create a new flow with the required bandwidth by building a bandwidth-satisfied multicast tree. The algorithm tries for minimization of the number of forwarders for the new flow. Bandwidthsatisfied multicasting protocol) has greater receiving ratios than MCEDAR, maintaining high receiving ratios (between 0.859 and 1) even when the network traffic reaches saturation. It admits more multicast groups than MCEDAR, if the network traffic is not saturated (the number of multicast groups is smaller than 5).

\subsection{QoS Multilayered Multicast Routing Protocol (QMMRP)}

Two algorithms Multiple Shortest Path Tree (MSPT) and Multiple Steiner Minimum Tree (MSMT) for multiple layer video multicasting in heterogeneous wireless ad hoc networks are detailed in [22]. The number of video layers which can be received and transmitted defines the capacity of a node. Nodes possessing capacity of one means they are capable of receiving and transmitting only one video layer if they are forwarding nodes or able to display the video if they are destination nodes. Factors limiting the capacity of a node are number of sessions participating in, remaining power, type of node and buffer size. More the available bandwidth more is the quantity of video layers that can be managed by an arbitrary node as it is not dependent only on its own capacity. QMMRP is a multicast routing protocol used on demand. Various multicast trees are constructed whenever the multicast source node has a transmission ready multicast video. The objective of QMMRP is to construct multiple multicast trees to maximize user's satisfaction. Shortest path tree (SPT) builds a multicast tree with minimum available path from source to destination. SPT ensures minimization of end-to-end delay from the multicast source to destination. Even though there are many resources in the network, existence of single SPT meeting destinations' requirement is difficult. Therefore, MSPT increases the quantity of video layers being delivered to each destination. Steiner minimum tree (SMT) algorithm builds a multicast tree that covers all the members of multicast group with lesser number of links. SMT ensures restriction on end-to-end delay of the multicast tree. MSMT are built keeping SMT and the video descriptions assignment in mind, very similar to MSPT. The developed algorithms result in increase of user satisfaction.

\subsection{Ad-hoc Mesh-based on-demand Multicast Routing Protocol with Quality of Service Support (AMOMQ)}

A new method for support of QoS routing with the use of estimation of required and available bandwidth, AMOMQ (Ad-hoc Mesh-based On-demand Multicast Routing Protocol with Quality of Service Support), is developed by authors in [23]. It implements two efficient optimization mechanisms for reduction of the control packets overhead generated during the route discovery process and for applying admission control to traffic. One of these methods finds its application in nodes which do not support QoS requirements and the other is for all intermediate nodes. While executing admission control in an intermediate node, the available bandwidth of the nearby nodes should be considered. Information about neighbourhood is very much critical for AMOMQ since it gives knowledge about the mobility, traffic and local topology. It is more critical for measurement of traffic, detection and recovery after route

APTIKOM J. CSIT Vol. 2, No. 1, $2017: 31-46$ 
failure. Each node periodically dispatches a "Hello" packet to broadcast its existence and traffic information to its nearby set. Whenever there is a requirement for establishment of route to any other node, the source disseminates a RREQ that contains the requested bandwidth, delay and node's neighbour list. Decision regarding admission is carried out at the processing node and its neighbouring table. Once the routes are constructed, the source is permitted to communicate with multicast groups using the selected routes and forwarding nodes. Here, soft state is used for maintenance of route. RREQ packet along with route setup procedure is sent frequently by each source to refresh or repair broken routes.

\subsection{QoS Constrained Multicast Routing For Mobile Ad Hoc Networks (QoS-MAODV-2LQoS)}

It becomes difficult for an application to know about the state of a network because of the significant utilization of resources and the changing nature of ad hoc network. A QoS Constrained Multicast Routing for Mobile Ad Hoc Networks (QoS-MAODV-2LQoS) is addressed in [24] that uses two QoS metrics: application layer metrics and network layer metrics. The aim of network layer metrics is to restrict the network resources and use the balanced network utilization. The path discovery procedure uses network layer metrics such as buffer level, power level, hop count and stability level to compute the state of QoS. For path selection procedure, the application layer metrics employed are delay, throughput and cost. The path discovery is initiated by the source with the broadcasting of a Route request (RREQ) along with the QoS extension to destination. The path selection procedure is initiated as soon as the path request message, sent by QoS, reaches the destination node. Based on class and state of QoS, the destination node chooses the path. For class=1, the path with the lesser end-to-end delay is chosen. For class $=2$, the path with the highest available bandwidth is chosen. For class $=3$, the shortest path is chosen. Then, Route Reply (RREP) is sent by the destination to the source. The queue gets prioritized service according to its weight and therefore, even application with lowest priority gets serviced.

\section{Hybrid Routing Strategy Based Multicast Routing Protocols \\ 7.1. QoS Multicasting Routing Protocol (QMR)}

Now a days, multimedia and group-oriented computing plays a crucial role for the users of MANETs. So QoS parameters become essential to support real time application and multimedia. In order to provide mobility to multimedia users, in [11] authors proposed a flexible hybrid scheme QoS multicasting routing protocol (QMR). The mechanisms of QMR provide fix reservation and shared reservation bandwidth in order to ensure QoS multicasting routing. For providing QoS multicasting routing from source to destinations, QMR uses forward nodes (FNs) and ensure load balancing inside the network. QMR is a mesh based routing protocol that establishes a QoS path from source node to destinations and provides bandwidth reservation for multicast group.

\subsection{Lantern-Tree-based QoS Multicast (LTM)}

The aim of QoS multicast routing protocol is to search for a multicast tree from source to destination in which the total bandwidth on the paths which are available is more than the minimal bandwidth required. A lantern-tree based QoS multicast protocol which includes a reliable mechanism for adoption of the CDMA-over-TDMA channel model by the MAC sub-layer, is proposed in [25]. Here we identify a lantern-tree to satisfy certain bandwidth requirements and moreover, the proposed model offers simple reliable mechanism to guarantee reliable communications. A path where each component is a lantern is called the lantern-path. In a lantern, each sub-path requires a sub-bandwidth. The number of sub-paths of a lantern depends on the network bandwidth. Lantern naturally increases the success rate of identification of a QoS route by providing a robust mechanism. LTM Protocol consists of three phases lantern identification, lantern-tree construction and lantern-tree maintenance. The second phase builds the lantern-tree by combining and joining lantern-paths from a source to all the available destinations. The last phase maintains the lantern for enhancement of the robustness and therefore preserves its stability.

\subsection{Hypercube-based Virtual Dynamic Backbone (HVDB)}

The presence of limited bandwidth between the mobile nodes and high dynamic topology complicates the design of QoS multicast routing protocols. Due to the nodes being mobile and less availability of resources like node energy and wireless link bandwidth, providing QoS guarantee in MANETs becomes more difficult. Authors in [26] proposed Hypercube-based Virtual Dynamic Backbone (HVDB) model for QoS-aware multicast communications that offers high availability and good load balancing properties in large-scale MANETs. HVDB comes from n-dimensional hyper cubes which 
contain properties like high small diameter, fault tolerance, symmetry and regularity. Mobility prediction and location-based clustering technique is used by this model to form stable clusters. It elects a Mobile Node as Cluster Head if it has the highest probability and the distance is minimal from the center of the cluster. It proposes a three tier structure: the Mobile Node Tier (MNT), the Hypercube Tier (HT) and the Mesh Tier (MT). MNT consists of Mobile Nodes that are grouped into clusters. Each MN can determine a circle called Virtual Circle (VC) where it stores the location information and the center of circle is called Virtual Circle Center (VCC). The HT contains many logical k dimensional hyper cubes whose nodes are originally Cluster Heads $(\mathrm{CH})$. The MT is a two-dimensional mesh network with each k-dimensional hypercube being a singular mesh node. HVDB contains some non-virtual and non-dynamic properties similar to stability and reality properties of the Internet backbone. It best describes the proactive local logical route maintenance algorithm, the summary-based membership update algorithm, and the logical location-based multicast routing algorithm.

\subsection{Hybrid QoS Multicast Routing Protocol (HQMRP)}

In [27], Hybrid QoS Multicast Routing Protocol (HQMRP) for minimisation of end-to-end delay by employing a source-tree multicast distribution structure, is proposed. To overcome path break resulting from mobility, receiver-driven reconnection approach is used by HQMRP where the disconnected subtree is flushed at first and then the receivers join the group again. HQMRP chooses Integrated Services (IntServ) model for emulation of a connection-oriented and virtual circuit connection for each flow that is admitted to the network. The basic state information about availability of the bandwidth is maintained in every node. The source uses a QoS routing protocol for finding a feasible path to each destination with the help of this bandwidth information. HQMRP utilizes explicit control packets to propagate and maintain the QoS state. HQMRP's routing protocol follows a dedicated approach with periodic link-state to minimize end-to-end delay and to cope up with fast-changing topology. Two clusters, operating at different frequencies, are connected to each other with the help of a commonly shared node referred to as gateway. Link-state information is shared only within the cluster in order to avoid updating of the expensive global link-state.

\subsection{QoS Multicast Routing Protocol for Clustering Mobile Ad Hoc Network (QMRPCAH)}

Clustering problem of MANET is dependent upon the topology of the network, node's geographical location, connectivity, range of signal, node mobility and the relativity between nodes. A QoS multicast routing protocol for clustering mobile ad hoc networks (QMRPCAH), proposed in [28] provides QoS-sensitive routes in the network environment along with mobility in a flexible and scalable way. Every local node In QMRPCAH is responsible for broadcasting of the delay information (along with its outgoing links) to every other node. All the remaining nodes recompute their routing tables which are within their cluster, after reception of the information. The remote subscription method, in which each node takes part in subscription after entering a new domain, is used in handling mobility. The links violating the bandwidth constraint is deleted first so that the flooding message is kept out of all the violated links.

\subsection{Cluster-based QoS Multicast Routing Protocol (CQMRP)}

A cluster-based QoS aware multicast routing protocol (CQMRP) for mobile ad hoc network is discussed in [29] where the multicast routes are constructed in a distributed manner. Analysis shows that the control overhead generated by this QoS aware multicast routing scheme is less than the other related schemes. After simulation, result shows that the packet delivery ratio of the described model is better than HQMRP in higher mobility scenario and very much likely to adapt to topology changes.

\subsection{A QoS-Aware Multicast Routing Protocol for Multimedia Applications in Mobile Ad Hoc Networks (QMOST)}

Traditional multicasting routing protocols do not consider QoS parameters on links in the network as these protocols can not use the Steiner tree problem in this scenario. That's lead towards a NP complete problem. In [30], authors proposed QMOST that supports multiple-metric routing features in MANETs in order to calculate QoS parameters in polynomial time using source based optimisation heuristic mechanism. Before forwarding data packets in the network to the overlay terminal in a broadcasting medium, QMOST encapsulates the packets in unicast packets. It operates in collaboration with QoS link state protocol (QOLSR) in order to ensure QoS in multicast medium, congestion control and resource reservation. 


\subsection{Location-based multicast routing for mobile ad hoc networks (LACMQR)}

The availability of tiny, less expensive, less-power GPS receiver and methods for the calculation of similar coordinates basing on the strength of the signal makes it easy for application of position-based routing algorithm. A loop-free distributed cluster multicast QoS routing protocol is detailed in [31] where the only requirement is that every node maintains its local state and with the help of the physical location information, it discovers and maintains the route. The total network is divided into clusters. In each cluster, with the use of a cluster head selection algorithm a cluster head is selected, after which gateways are chosen by gateway selection algorithm. Each node carries on self-clustering with the help of position information of each node. When a source node calls for transmission of packets but a valid route is not found, a route discovery procedure for setting up of a path is initiated. A route probe packet, called PROBE, is sent to its cluster head and is then further forwarded to its gateway. The destination node chooses an optimal route by following the best predecessor replacement policy and replies back with an acknowledgement packet to its predecessor, mentioned as ACK. After the ACK packet is received by the source node, route establishment is done and thus, data packets are transmitted by the source node. When a packet encounters a broken link, the node communicates the source node by sending a backward ERR packet. After the ERR packet is received by the source node, deletion of the related entry of routing table and initiation of route discovery for reconstruction of new path is carried out.

\section{Clustering Based Routing Protocols}

\subsection{Weight Based Clustering Protocols}

A distributed weighted clustering approach for improvisation of QoS for MANETs that use a combined weight metric is proposed in [32]. Various system parameters that are taken into account here are transmission range, ideal node degree, mobility and energy of the nodes. The determination of cluster head is done with the help of some combinations of these parameters. Authors in [33] have developed an architecture consisting of service discovery relying mainly on cluster techniques which carries on the selection of cluster head by allocation of a combined weight value based on the factors like Power Level, Connectivity and Stability, which are originally meant for wireless mobile ad hoc networks. The main requirements for service discovery system in MANETs are Optimal Service, Selection and Robustness Faces to mobility. It infers that most of the existing discovery mechanisms do not take into account these issues or some of them consider these issues partially. Their solution involves a cluster based approach, where the cluster head nodes form a distributed service registry.

A Flexible Weight Based Clustering Algorithm (FWCA) for MANETs, proposed in [34] reviews many existing algorithms like the Highest Degree, the Lowest Identifier, Distributed Clustering Algorithm and many more. In order to give each node a unique ID, this protocol uses the MAC address. Each node in a cluster has complete information about its cluster head. Each cluster head keeps track of other cluster heads by maintaining a cluster head information table denoted as ' $\mathrm{CH}$ table' for the storage of information about the remaining cluster heads. In Adaptive Weight Cluster Based Routing algorithm [35], the cluster head is selected on weight basis where the weight is calculated for each node in cluster taking into consideration the factors like node's energy level, its stability and its connectivity. To search multiple paths for distribution of data packets between the nodes "hierarchical structure management" is used. The protocol aims in getting strong connectivity as well as scalability. In [36], Yu-Xuan Wang and Forrest Sheng Bao have proposed an Entropy-based Weight Clustering Algorithm [EWCA] and its optimization for Ad hoc networks which increases the stability of the network it improves the number of clusters and re affiliation frequency reducing network overhead and stabilizing the structure of the network. A flexible and robust Weight based Clustering Algorithm for MANETs to maintain local topology as well as for management of the nodes, is covered in [37].

Initially the nodes exchange NEIG_MSG with its neighbour for updating cluster information. Each node in the cluster checks for itself whether or not is it suitable to become the cluster head using clustering algorithm. A weight function is given which calculates the weight of the nodes and thus helps in selecting the cluster head. Cluster based Routing Protocol for Mobile Ad Hoc Networks (CRP), proposed in [38] aims at decreasing the packet delay, increasing the packet delivery ratio, increasing the tolerance of errors and fast routing. If a node in the route fails, the cluster head assigns its work to another node thus making the system error tolerant. It includes an election algorithm for calculation of the node's weight so that the node possessing higher weight can be elected as the cluster head. Distributed Score Based Clustering Algorithm (DSBCA) for MANETs [39] focuses at selecting the cluster head based on its residual battery power, Number of neighbours, Number of Members and Stability. In order to become a cluster head, each node calculates its own "score" and informs its neighbours about its score and selects

Quality of Service based Multicasting Routing Protocols for MANETs: A Survey (Prabhat Kumar Sahu) 
a cluster head from within its neighbours having the highest score in a distributed manner taking current updated scores of the nodes into considerations.

\subsection{Mobility Based Clustering Protocols}

Cross-CBRP discussed in [40] takes into account multiple layers such as physical, MAC and network layers. In cross layer design, information exchange takes place between different layers and according to this information, the total performance is optimized. In CBRP, each node sends a "Hello Message" to indicate its presence to other nearby nodes. Each node after reception of the Hello Message, carries on updating its neighbour tables and then compares its ID with its neighbour. If nodes find that its ID is the lowest among its neighbours, it becomes cluster head. In [41], a protocol that overcomes the problem of signal, power and bandwidth wastage by using Weighted clustering algorithm for formation of clusters and prediction of mobility for maintaining the cluster. The cluster is formed by sending beacon message by each node to notify each of its neighbours. The node possessing less weight is selected as the Cluster Head. The cluster maintenance is defined by the threshold property of battery power and the movement of the node. The cluster based protocol developed by authors in [42] focuses on the stability of the cluster as it guarantees a longer lifetime of the cluster being formed. It is capable of exactly predicting the mobility of every host due to the stability of its neighbourhood. This information is useful in creating cluster from hosts that will remain neighbours for a longer period of time. In this approach, a cluster head is elected for each cluster and a mobile host $(\mathrm{MH})$ is elected for inter-cluster communication. The main cause for change in topology in MANETs is the host mobility. Therefore this protocol acknowledges the importance of host mobility for building stable clusters.

\subsection{Location Based Clustering Protocols}

A protocol for routing in inter domain of MANETs is described in [43]. Border Gateway protocol is used for inter-domain routing in internet but is not applicable in MANETs because of its static nature. In the proposed protocol, clusters are formed according to group affinity. The cluster head acts as the local DNS for its own cluster and for the neighbouring clusters. The cluster head notifies its neighbours as well as the whole network about its connectivity, members and domain information. CLACR protocol presented in [44] uses the location information for routing where the whole network is divided into a number of Squares Clusters. The types of nodes here are stated as: Cluster Head (HD), Location Server (LS) and Backup Location Server (BLS). Then each cluster within the network selects its own cluster head (HD) using cluster head selection algorithm. Only the Cluster head (HD), Source node and Destination node participate in CLACR. And, the location server (LS) is selected by location server election algorithm. Location Enhanced Routing Protocol based on Cluster Based Routing Protocol (LECBRP) is proposed in [45] that uses smart antennas for route discovery by identifying the location of a node and thus making route decisions for clustered MANETs. A smart antenna is an array of antennas with signal processing algorithm used to identify signal structure. Smart antennas are more suitable for MANETs than global positioning system (GPS) as GPS are ineffective indoors, consumes very high power and has a less degree of precision as compared to smart antennas. ILCRP proposed in [46] can be used for both inter as well as intra cluster communications. It takes into account the information about clusters and also their location by using GPS. This protocol is segregated into three stages where first stage is for formation of cluster, second is for maintaining the cluster and the last stage is for route discovery. In ILCRP due to usage of GPS, the control overhead for route discovery is less. As the cluster head in this protocol knows the exact source location and destination nodes location in advance, therefore the packet is delivered accurately with less end to end delay.

\subsection{Artificial Intelligence Based Clustering Protocols}

A fuzzy relevance-based cluster head selection algorithm for wireless mobile ad-hoc sensor networks is addressed in [47]. The fuzzy information present in the mobile routing nodes helps in deciding and managing the cluster. Based on this very information, selection of Cluster head $(\mathrm{CH})$ and Cluster members (CM) is performed. Fuzzy relevance degree (FRD) with fuzzy value is used for this process. FRD is used to maintain the stability of the structure of the cluster which ultimately minimizes the changes in the structure and overhead caused due to clustered changes. Authors in [48] have proposed an Optimized Routing in Mobile Ad-Hoc Networks using Evolutionary Location Intelligence. In general cases, if we apply the positioning method, then it causes problem due to unstable nature of the network and if we apply greedy method, then about half of the messages at average degree of below 4, are not delivered. It means that these two methods cause problem in routing, but if we apply these two methods in combination, then these problems might get resolved. In this protocol, concave nodes are identified

APTIKOM J. CSIT Vol. 2, No. 1, $2017: 31-46$ 
because these nodes flood their neighbours and reject the further copies of same message. Routing Optimization using Genetic Algorithm in Ad Hoc Networks [49] puts emphasis on limiting the use of resources present inside or outside the node such as mobility, transmission power, degree and battery power. Cluster head Election for CGSR Routing Protocol Using Fuzzy Logic Controller for Mobile Ad Hoc Network is detailed in [50] that implements CL selection using a method of mixed geometry and resource usability.

\section{CONCLUSION}

In this paper, our focus has been on several multicasting routing protocols that ensure QoS provision in MANETs. Based on our suvey, all the design approach of many multicasting routing protocols focus on QoS parameters like bandwidth rather than other parameters like jitter, delay and others. Again scalability is one of the major issues in wireless network which is not considered by most of the protocols. Several QoS based multicasting routing protocols are proposed, but still multiple mechanisms that have not been investigated in this field. There fore in future it should be further research on heterogeneous and wireless network with respect to several QoS parameters and must be focused on QOS multicasting routing algorithms for multimedia applications to solve above said issues.

\section{References}

[1] Sinha, P.; Sivakumar, R.; Bharghavan, V. MCEDAR: Multicast Core-Extraction Distributed Ad Hoc Routing. In Proceedings of the IEEE Wireless Communications and Networking Conference, New Orleans, LA, USA, September 1999; pp. 1313-1317.

[2] Chen, H.; Sun, B.; Zeng, Y. QoS Multicast Routing Algorithm in MANET: An Entropy-Based GA. In Proceedings of the International Conference on Intelligent Computing, Kunming, China, August 2006; pp. 1279-1289.

[3] Takashima, E.; Murata, Y.; Shibata, N.; Yasumoto, K.; Ito, M. A Method for Distributed Computation of SemiOptimal Multicast Tree in MANET. In Proceedings of the 8th IEEE Wireless Communications and Networking Conference, Kowloon, China, March 2007.

[4] Yen, Y.; Chan, Y.; Chao, H.; Park, J.H. A genetic algorithm for energy-efficient based multicast routing on MANETs. Comput. Commun. 2008, 31, 2632-2641.

[5] Wang, H.; Shi, Z. An Ant Colony Algorithm Based on Orientation Factor for QoS Multicast Routing in Ad Hoc Networks. In Proceedings of the 2008 Third International Conference on Communications and Networking in China, Hangzhou, China, August 2008; pp. 321-326.

[6] Pagani E.; Rossi G.P. A Framework for the Admission Control of QoS Multicast Traffic in Mobile Ad Hoc Networks. In Proceedings of the 4th ACM international workshop on Wireless mobile multimedia, Rome, Italy, 2001; pp. 2-11.

[7] Guo, S.; Yang, O. QoS-aware minimum energy multicast tree construction in wireless ad hoc networks. Ad Hoc Netw. 2004, 2, 217-229.

[8] Ng, J.M.; Low, C.P.; Teo, H.S. On-Demand QoS Multicast Routing and Reservation Protocol for MANETs. In Proceedings of the 2004 IEEE 15th International Symposium on Personal, Indoor and Mobile Radio Communications, Barcelona, Spain, September 2004; pp. 2504-2508.

[9] Brahim, I.B.; Jemaa, M.B. Multicast Routing Protocol for Ad Hoc Networks with a Quality of Service $<$ Qos$A O D V>>$. In Proceedings of the IEEE International Conference on Electronics, Circuits, and Systems, Gammarth, Tunisia, December 2005.

[10] Bür, K.; Ersoy, C. Ad hoc quality of service multicast routing. Comput. Commun. 2005, 29, 136-148.

[11] Saghir, M.; Wan, T.C.; Budiarto R. Load Balancing QoS Multicast Routing Protocol in Mobile Ad Hoc Networks. In Lecture Notes in Computer Science; Cho, K., Jacquet, P., Eds.; Springer-Verlag: Berlin, Germany, 2005; Volume 3837/2005, pp. 83-97.

[12] Tebbe, H.; Kassler, A.J.; Ruiz, P.M. QoS-Aware Mesh Construction to Enhance Multicast Routing in Mobile Ad Hoc Networks. In Proceedings of the First International Conference on Integrated Internet Ad Hoc and Sensor Networks, Nice, France, May 2006.

[13] Saghir, M.; Wan, T.; Budiarto, R. QoS Multicast Routing Based on Bandwidth Estimation in Mobile Ad Hoc Networks. In Proceedings of the International Conference on Computer and Communication Engineering, Kuala Lumpur, Malaysia, May 2006; pp. 9-11.

[14] Yang, M.; Layuan, L.; Fang, Y. Secure Efficient QoS Multicast Route Discovery for MANET. In Proceedings of the First International Conference on Communications and Networking in China, Beijing, China, October 2006; pp. 1-5.

[15] Saghir, M.; Wan, T.; Budiarto, R. A new cross-layer framework for QoS multicast applications in mobile ad hoc networks. IJCSNS 2006, 6, 142-151.

[16] Darehshoorzadeh, A.; Dehghan, M.; Motlagh, M.R.J. Quality of service support for ODMRP multicast routing in ad hoc networks. Lect. Note. Comput. Sci. 2007, 4686, 237-247. 
[17] Promkotwong, D.; Ohm S. A Mesh-Based QoS Aware Multicast Routing Protocol. In Proceedings of the 8th ACIS International Conference on Software Engineering, Artificial Intelligence, Networking, and Parallel/Distributed Computing, Qingdao, China, 31 July-1 August 2007; pp. 1046-1051.

[18] Wu, H.; Jia, X. QoS multicast routing by using multiple paths/trees in wireless ad hoc networks. Ad Hoc Netw. $2007,5,600-612$.

[19] Vida Lashkari, B.O.; Dehghan, M. QoS-aware Multicast Ad Hoc On-Demand Distance Vector Routing. In Proceedings of the World Congress on Engineering, London, UK, July 2007; pp. 1506-1511.

[20] Chen, Y.; Lin, T.; Lin, Y. A hexagonal-tree TDMA-based QoS multicasting protocol for wireless mobile ad hoc networks. Telecommun. Syst. 2007, 35, 1-20.

[21] $\mathrm{Hu}, \mathrm{C}$; $\mathrm{Wu}$, E.H.-K.; Chen, G. Bandwidth-satisfied multicast trees in MANETs. IEEE Trans. Mob. Comput. 2008, 7, 712-723.

[22] Badarneh, O.; Kadoch, M.; Elhakeem, A. QoS multilayered multicast routing protocol for video transmission in heterogeneous wireless ad hoc networks. WSEAS Trans. Comput. 2008, 7, 680-693.

[23] Nourazar, S.; Dehghan, M. AMOMQ: Ad-Hoc Mesh-Based On-demand Multicast Routing Protocol with Quality of Service Support. In Proceedings of the 2009 International Association of Computer Science and Information Technology—Spring Conference, Singapore, April 2009; pp. 95-99.

[24] Latha, P.; Ramachandran, R. QoS Constrained Multicast Routing for Mobile Ad Hoc Networks. IJCSNS 2009, 9, 66-70.

[25] Chen, Y.; Ko, Y. A lantern-tree-based QoS on-demand multicast protocol for a wireless mobile ad hoc network. IEICE Trans. Commun. 2004, E87-B, 717-726.

[26] Wang, G.; Cao, J.; Zhang, L.; Chan, K.C.C.; Wu, J. A Novel QoS Multicast Model in Mobile Ad Hoc Networks. In Proceedings of the 19th IEEE international Parallel and Distributed Processing Symposium (Ipdps'05)— Workshop 8, Denver, CO, USA, April 2005.

[27] Wang, W.; Soong, B.; Chew, J. A Novel Protocol of QoS Multicasting for Large Scale MANET. In Proceedings of the First IEEE Conference on Industrial Electronics and Applications, Singapore, May 2006; pp. 79-84.

[28] Chunlin, L.; Layuan, L. A QoS multicast routing protocol for clustering mobile ad hoc networks. Comput. Commun. 2007, 30, 1641-1654.

[29] Nargunam, A.S.; Sebastian, M.P. QoS-aware multicast routing for mobile ad hoc networks. Int. J. Bus. Data Commun. Netw. 2008, 4, 1-21.

[30] Hakim, B. A QoS-Aware Multicast Routing Protocol for Multimedia Applications in Mobile Ad Hoc Networks. In Proceedings of the 11th ACM International Conference on Modeling, Analysis, and Simulation of Wireless and Mobile Systems, Vancouver, Canada, October 2008; pp. 244-251.

[31] Shih, T.; Shih, C.; Chen, C. Location-based multicast routing protocol for mobile ad hoc networks. WSEAS Transac. Comput. 2008, 7, 1270-1279.

[32] Naveen Chauhan, L. K. Awasthi, Narottam chand, Vivek Katiyar, and Ankit Chug, "A Distributed Weighted Cluster Based Routing Protocol for Manets," World Academy of Science, Engineering and Technology 75, pp.1167-1171, 2011.

[33] S. Karunakaran and P. Thangaraj, "A Cluster-Based Service Discovery Protocol for MobileAd-hoc Networks," American Journal of Scientific Research, Issue 11, pp.179-190, 2011.

[34] Zouhair El-Bazzal, Michel Kadoch, and Basile L. Agba, François Gagnon and Maria Bennani, "A Flexible Weight Based Clustering Algorithm in Mobile Ad hoc Networks".

[35] S. Karunakaran and DR. P. Thangaraj, "An Adaptive Weighted Cluster Based Routing (AWCBRP) Protocol for Mobile Adhoc Networks".

[36] Y-X. Wang and Forrest Sheng Bao, "An Entropy-based Weighted Clustering Algorithm and Its Optimization for Ad Hoc Networks".

[37] R. Pandi Selvam and V. Palanisamy, "Stable and Flexible Weight based Clustering Algorithm in Mobile Ad hoc Networks,"International Journal of Computer Science and Information Technologies, Vol. 2 (2), pp.824$828,2011$.

[38] M. Rezaee and M. Yaghmaee, "Cluster based Routing Protocol for Mobile Ad Hoc Networks".

[39] Sahar Adabi, Sam Jabbehdari, Amirmasoud Rahmani, and Sepideh Adabi, "A Novel Distributed Clustering Algorithm for Mobile Ad-hoc Networks," Journal of Computer Science, vol. 4, issue: 2, pp.161-166,2008.

[40] Arash Dana, Amir Mansour Yadegari, Marzieh Hajhosseini, and Tina Mirfakhraie, "A Robust Cross-Layer Design of Clustering-Based Routing Protocol for MANET,” pp. 1055-1059.ICACT, 2008.

[41] S. Muthuramalingam, R. Raja Ram, Kothai Pethaperumal, and V.Karthiga Devi, "A Dynamic Clustering Algorithm for MANETs by modifying Weighted Clustering Algorithm with Mobility Prediction". International Journal of Computer and Electrical Engineering, Vol.2, No. 4, pp.709-714, August, 2010.

[42] Charalampos Konstantopoulos, Damianos Gavalas, and Grammati Pantziou, "Clustering in mobile ad hoc networks through neighborhood stability-based mobility prediction," pp.1727-1824,2008

[43] Biao Zhou, Zhen Cao, and Mario Gerla, "Cluster-based Inter-domain Routing (CIDR) Protocolfor MANET's".

[44] T-F. Shih and H-C. Yen, "Core Location-Aided Cluster-Based Routing Protocol for Mobile Ad Hoc Networks,” pp223-228, 2006.

[45] Hatem Hamad, Abdessalam Elhabbash, Fuad Abuowaimer, and Yousif Mansour, "Location Enhanced Cluster Based Routing Protocol," pp.1-14.

APTIKOM J. CSIT Vol. 2, No. 1, 2017 : $31-46$ 
[46] S. Mangai and A. Tamilarasi "A new approach to Geographic Routing for Location aided Cluster based MANETs," pp.1-22.

[47] Chongdeuk Lee and Taegwon Jeong, "FRCA: A Fuzzy Relevance-Based Cluster Head Selection Algorithm for Wireless Mobile Ad-Hoc Sensor Networks".

[48] J. Arunadevi and V. Rajamani, "Optimized routing in Mobile Ad Hoc Networks using Evolutionary Location Intelligence," IJCA Special Issue on "Mobile Ad-hoc Networks" MANETs, 2010.

[49] Mustafa Al-Ghazal and Ayman El-Sayed,Hamedy Kelash, "Routing Optimization using Genetic Algorithm in Ad Hoc Networks," pp.505-511,Dec 2007.

[50] K. Venkata Subbaiah and M. M. Naidu, "Cluster head Election for CGSR Routing Protocol Using Fuzzy Logic Controller for Mobile Ad Hoc Network," Int. J. of Advanced Networking and Applications, Vol: 01, Issue: 04, Pages: 246-251, 2010.

\section{Appendix}

Table Comparison of QoS multicast routing protocols in MANETs.

\begin{tabular}{|c|c|c|c|c|c|c|c|c|}
\hline No. & Protocol & $\begin{array}{l}\text { Routing } \\
\text { Scheme }\end{array}$ & QoS Parameters & $\begin{array}{l}\text { Multicast } \\
\text { Distribution }\end{array}$ & $\begin{array}{l}\text { MAC } \\
\text { sublayer }\end{array}$ & $\begin{array}{l}\text { Performance } \\
\text { Matrics }\end{array}$ & $\begin{array}{l}\text { Group } \\
\text { creations }\end{array}$ & $\begin{array}{l}\text { Failure } \\
\text { handling }\end{array}$ \\
\hline 1 & MCEDAR[1] & Proactive & bandwidth & Hybrid & Any & $\begin{array}{l}\text { Average latency, } \\
\text { Control Overhead }\end{array}$ & Soft & $\begin{array}{l}\text { Common } \\
\text { core }\end{array}$ \\
\hline 2 & EQMGA[2] & Proactive & bandwidth & SST & Any & $\begin{array}{l}\text { PDR, Average } \\
\text { latency }\end{array}$ & Source & - \\
\hline 3 & HQMGA[3] & Proactive & bandwidth & SST & 802.11 & Throughput & Source & - \\
\hline 4 & $\mathrm{EGA}[4]$ & Proactive & Delay & SST & Any & - & Source & - \\
\hline 5 & $\mathrm{MACO}[5]$ & Proactive & $\begin{array}{l}\text { Bandwidth,Dela } \\
\text { y,Jitter }\end{array}$ & SST & Any & $\begin{array}{l}\text { PDR, Average } \\
\text { latency }\end{array}$ & ource & - \\
\hline 6 & M-CAMP[6] & Reactive & bandwidth & Any kind & Any & $\begin{array}{l}\text { Member and Session } \\
\text { Satisfaction Grade }\end{array}$ & Any kind & Local \\
\hline 7 & QOS-MEM[7] & Reactive & bandwidth & SST & TDMA & Throughput & Source & - \\
\hline 8 & ODQMM[8] & Reactive & bandwidth & ST & TDMA & $\begin{array}{l}\text { PDR, Average } \\
\text { latency }\end{array}$ & Receiver & Local \\
\hline 9 & QoS-AODV[9] & Reactive & Delay & ST & Any & $\begin{array}{l}\text { Member and Session } \\
\text { Satisfaction Grade }\end{array}$ & Receiver & Soft \\
\hline 10 & AQM[10] & Reactive & bandwidth & ST & Any & $\begin{array}{l}\text { Member and Session } \\
\text { Satisfaction Grade }\end{array}$ & Receiver & Global \\
\hline 11 & QMR[11] & Hybrid & bandwidth & Mesh & 802.11 & $\begin{array}{l}\text { Throughput } \\
\text { PDR, Control }\end{array}$ & Source & - \\
\hline 12 & QAMNET[12] & Reactive & bandwidth & Mesh & 802.11 & $\begin{array}{l}\text { Overhead, Average } \\
\text { latency }\end{array}$ & Source & Soft \\
\hline 13 & E-QMR[13] & Reactive & bandwidth & Mesh & 802.11 & $\begin{array}{l}\text { PDR, Control } \\
\text { Overhead, Average } \\
\text { latency }\end{array}$ & Source & Soft \\
\hline 14 & SEQMRAN[14] & Reactive & $\begin{array}{l}\text { Bandwidth,Dela } \\
\text { y,Jitter }\end{array}$ & ST & 802.11 & $\begin{array}{l}\text { Average latency, } \\
\text { Control Overhead } \\
\text { PDR, Control }\end{array}$ & Source & - \\
\hline 15 & FQM[15] & Reactive & bandwidth & Mesh & 802.11 & $\begin{array}{l}\text { Overhead, Average } \\
\text { latency }\end{array}$ & Source & Soft \\
\hline 16 & $\begin{array}{l}\text { QoS- } \\
\text { ODMRP[16] }\end{array}$ & Reactive & bandwidth & Mesh & 802.11 & $\begin{array}{l}\text { PDR, Traffic } \\
\text { Admission Ratio }\end{array}$ & Source & Soft \\
\hline 17 & QMRP[17] & Reactive & bandwidth & Mesh & 802.11 & $\begin{array}{l}\text { PDR, Control } \\
\text { Overhead }\end{array}$ & Source & $\begin{array}{l}\text { Soft+Loca } \\
1\end{array}$ \\
\hline 18 & MPT[18] & Reactive & bandwidth & SST & $\begin{array}{l}\text { CDMA } \\
\text { over } \\
\text { TDMA }\end{array}$ & $\begin{array}{l}\text { Success Ratio, } \\
\text { Network } \\
\text { Resources Cost }\end{array}$ & Source & Global \\
\hline 19 & $\begin{array}{l}\text { QoS- } \\
\text { MADOV[19] }\end{array}$ & Reactive & $\begin{array}{l}\text { Bandwidth,Dela } \\
\text { y,Jitter }\end{array}$ & ST & 802.11 & $\begin{array}{l}\text { PDR, Control } \\
\text { Overhead, Average } \\
\text { latency }\end{array}$ & Receiver & Soft \\
\hline 20 & HTQ[20] & Reactive & bandwidth & Any & TDMA & $\begin{array}{l}\text { Average latency, } \\
\text { Control Overhead }\end{array}$ & Source & Backup \\
\hline 21 & HU[21] & Reactive & bandwidth & & - & - & - & - \\
\hline 22 & QMMRP[22] & Reactive & bandwidth & SST & $\begin{array}{l}\text { CDMA } \\
\text { over } \\
\text { TDMA }\end{array}$ & $\begin{array}{l}\text { PDR, Control } \\
\text { Overhead, Average } \\
\text { latency }\end{array}$ & Source & Local \\
\hline 23 & AMOMQ[23] & Reactive & bandwidth & Mesh & 802.11 & $\begin{array}{l}\text { RREQ Control Load } \\
\text { Packet,PDR, } \\
\text { Average latency }\end{array}$ & Source & Soft \\
\hline 24 & $\begin{array}{l}\text { QoS-MADOV- } \\
\text { 2LQoS[24] }\end{array}$ & Reactive & $\begin{array}{l}\text { Bandwidth,Dela } \\
\text { y,Jitter }\end{array}$ & ST & 802.11 & $\begin{array}{l}\text { PDR, Control } \\
\text { Overhead, Average } \\
\text { latency }\end{array}$ & Receiver & Soft \\
\hline 25 & LTM[25] & Hybrid & bandwidth & Any & $\begin{array}{l}\text { CDMA } \\
\text { over }\end{array}$ & $\begin{array}{l}\text { Success Rate, } \\
\text { Overhead, Average }\end{array}$ & Source & soft \\
\hline
\end{tabular}




\begin{tabular}{|c|c|c|c|c|c|c|c|c|}
\hline & & & & & TDMA & Latency & & \\
\hline 26 & HVDB[26] & Hybrid & $\begin{array}{l}\text { Bandwidth,Dela } \\
\text { y }\end{array}$ & SST & Any & Success Ratio & Source & Soft \\
\hline 27 & HQMRP[27] & Hybrid & bandwidth & SST & TDMA & $\begin{array}{l}\text { Average latency, } \\
\text { Control Overhead }\end{array}$ & Source & Local \\
\hline 28 & QMRPCAH[28] & Hybrid & $\begin{array}{l}\text { Bandwidth,Dela } \\
\text { y }\end{array}$ & SST & MACA & $\begin{array}{l}\text { Success Rate of } \\
\text { Joining } \\
\text { Request, Message } \\
\text { Overhead, PDR, } \\
\text { Bandwidth }\end{array}$ & Receiver & Global \\
\hline 29 & CQMRP[29] & Hybrid & $\begin{array}{l}\text { Bandwidth,Dela } \\
\mathrm{y}, \text { Jitter }\end{array}$ & SST & Any & $\begin{array}{l}\text { Average latency, } \\
\text { Control Overhead }\end{array}$ & Source & Local \\
\hline 30 & QMOST[30] & Hybrid & bandwidth & ST & 802.11 & $\begin{array}{l}\text { PDR, Control } \\
\text { Overhead }\end{array}$ & Source & Global \\
\hline 31 & LACMQR[31] & Hybrid & bandwidth & SST & Any & $\begin{array}{l}\text { Average latency, } \\
\text { Control Overhead }\end{array}$ & Source & - \\
\hline 32 & FWCA & $\begin{array}{l}\text { Weighted } \\
\text { cluster-based }\end{array}$ & $\begin{array}{l}\text { End-to-End } \\
\text { Delay, } \\
\text { Success Ratio, }\end{array}$ & - & - & - & -- & - \\
\hline 33 & EWCA & $\begin{array}{l}\text { Weighted } \\
\text { cluster-based }\end{array}$ & $\begin{array}{l}\text { Average } \\
\text { number of } \\
\text { clusters, } \\
\text { Average } \\
\text { transition } \\
\text { number on each } \\
\mathrm{CH} \text {, Average } \\
\text { number of } \mathrm{CH} \\
\text { changes, } \\
\text { Re-affiliation } \\
\text { count }\end{array}$ & - & - & - & -- & - \\
\hline 34 & CRP & $\begin{array}{l}\text { Weighted } \\
\text { cluster-based }\end{array}$ & $\begin{array}{l}\text { Control } \\
\text { overhead, } \\
\text { Average end-to } \\
\text { end delay, } \\
\text { Average Packet } \\
\text { Delivery Ratio } \\
\text { End-to-End }\end{array}$ & - & - & - & -- & - \\
\hline 35 & DSBCA & $\begin{array}{l}\text { Weighted } \\
\text { cluster-based }\end{array}$ & $\begin{array}{l}\text { Delay, } \\
\text { Success Ratio, } \\
\text { Overhead }\end{array}$ & - & - & - & -- & - \\
\hline 36 & Cross-CBRP & $\begin{array}{l}\text { Mobility } \\
\text { cluster-based }\end{array}$ & $\begin{array}{l}\text { Packet Delivery } \\
\text { Ratio, } \\
\text { Throughput, } \\
\text { Overhead } \\
\text { Packets, End-to- } \\
\text { end Delay }\end{array}$ & - & - & - & -- & - \\
\hline 37 & CLACR & $\begin{array}{l}\text { Location } \\
\text { cluster-based }\end{array}$ & $\begin{array}{l}\text { Packet Delivery } \\
\text { Ratio }\end{array}$ & - & - & - & -- & - \\
\hline 38 & LECBRP & $\begin{array}{l}\text { Location } \\
\text { cluster-based }\end{array}$ & $\begin{array}{l}\text { Route } \\
\text { construction } \\
\text { success ratio, } \\
\text { Route set up } \\
\text { time, } \\
\text { Route life time, } \\
\text { Data delivery } \\
\text { rate. }\end{array}$ & - & - & - & -- & - \\
\hline 39 & ILCRP & $\begin{array}{l}\text { Location } \\
\text { cluster-based }\end{array}$ & $\begin{array}{l}\text { End to End } \\
\text { Delay, } \\
\text { Packet Delivery } \\
\text { Ratio, Control } \\
\text { Overhead }\end{array}$ & - & - & - & -- & - \\
\hline 40 & CGSR & $\begin{array}{l}\text { Artificial } \\
\text { Intelligence } \\
\text { cluster-based }\end{array}$ & $\begin{array}{l}\text { Re affiliations } \\
\text { per unit time, } \\
\text { Average } \\
\text { Number of } \\
\text { Clusters, Load } \\
\text { balance } \\
\text { factor, } \\
\text { cumulative } \\
\text { distribution }\end{array}$ & - & - & - & -- & - \\
\hline
\end{tabular}

APTIKOM J. CSIT Vol. 2, No. 1, 2017 : 31 -46 